\title{
ON THE LARGEST PRIME FACTOR OF THE MERSENNE NUMBERS
}

\author{
KEVIN FORD, FLORIAN LUCA and IGOR E. SHPARLINSKI ${ }^{\bowtie}$
}

(Received 7 August 2008)

\section{Abstract}

Let $P(k)$ be the largest prime factor of the positive integer $k$. In this paper, we prove that the series

$$
\sum_{n \geq 1} \frac{(\log n)^{\alpha}}{P\left(2^{n}-1\right)}
$$

is convergent for each constant $\alpha<1 / 2$, which gives a more precise form of a result of C. L. Stewart ['On divisors of Fermat, Fibonacci, Lucas and Lehmer numbers', Proc. London Math. Soc. 35(3) (1977), 425-447].

2000 Mathematics subject classification: primary 11B83, 11N25.

Keywords and phrases: primes, Mersenne numbers, applications of sieve methods.

\section{Main result}

Let $P(k)$ be the largest prime factor of the positive integer $k$. The quantity $P\left(2^{n}-1\right)$ has been investigated by many authors (see [1, 3, 4, 10-12, 14-16]). For example, the best-known lower bound

$$
P\left(2^{n}-1\right) \geq 2 n+1 \quad \text { for } n \geq 13
$$

is due to Schinzel [14]. No better bound is known even for all sufficiently large values of $n$.

Stewart $[15,16]$ gave better bounds provided that $n$ satisfies certain arithmetic or combinatorial properties. For example, he showed in [16], and this was also proved independently by Erdôs and Shorey [4], that

$$
P\left(2^{p}-1\right)>c p \log p
$$

holds for all sufficiently large prime numbers $p$, where $c>0$ is an absolute constant and $\log$ is the natural logarithm. This was an improvement upon a previous result of

(C) 2009 Australian Mathematical Society 0004-9727/2009 \$16.00 
his from [15] with $(\log p)^{1 / 4}$ instead of $\log p$. Several more results along these lines are presented in Section 3.

Here, we continue to study $P\left(2^{n}-1\right)$ from a point of view familiar to number theory which has not yet been applied to $P\left(2^{n}-1\right)$. More precisely, we study the convergence of the series

$$
\sigma_{\alpha}=\sum_{n \geq 1} \frac{(\log n)^{\alpha}}{P\left(2^{n}-1\right)}
$$

for some real parameter $\alpha$.

THEOREM 1. The series $\sigma_{\alpha}$ is convergent for all $\alpha<1 / 2$.

The rest of the paper is organized as follows. We introduce some notation in Section 2. In Section 3 we comment on why Theorem 1 is interesting and does not immediately follow from already known results. In Section 4 we present a result due to Stewart [16] which plays a crucial role in our argument. Finally, in Section 5, we give a proof of Theorem 1 .

\section{Notation}

In what follows, for a positive integer $n$, we use $\omega(n)$ for the number of distinct prime factors of $n, \tau(n)$ for the number of divisors of $n$ and $\varphi(n)$ for the Euler function of $n$. We use the Vinogradov symbols $\gg, \ll$ and $\asymp$ and the Landau symbols $O$ and $o$ with their usual meaning. The constants implied by them might depend on $\alpha$. We use the letters $p$ and $q$ to denote prime numbers. Finally, for a subset $\mathcal{A}$ of positive integers and a positive real number $x$, we write $\mathcal{A}(x)$ for the set $\mathcal{A} \cap[1, x]$.

\section{Motivation}

Stewart [16] proved the following two statements:

A. If $f(n)$ is any positive real-valued function which is increasing and $f(n) \rightarrow \infty$ as $n \rightarrow \infty$, then the inequality

$$
P\left(2^{n}-1\right)>\frac{n(\log n)^{2}}{f(n) \log \log n}
$$

holds for all positive integers $n$ except for those in a set of asymptotic density zero.

B. Let $\kappa<1 / \log 2$ be fixed. Then the inequality

$$
P\left(2^{n}-1\right) \geq C(\kappa) \frac{\varphi(n) \log n}{2^{\omega(n)}}
$$

holds for all positive integers $n$ with $\omega(n)<\kappa \log \log n$, where $C(\kappa)>0$ depends on $\kappa$. 
Since for every fixed $\varepsilon>0$ we have

$$
\sum_{n \geq 2} \frac{\log \log n}{n(\log n)^{1+\varepsilon}}<\infty
$$

assertion A above, taken with $f(n)=(\log n)^{\varepsilon}$ for some fixed small positive $\varepsilon<1-\alpha$, motivates our Theorem 1. However, since Stewart [16] gives no analysis of the exceptional set in assertion $\mathbf{A}$ (that is, of the size of the set of numbers $n \leq x$ such that the corresponding estimate fails for a particular choice of $f(n))$, this alone does not lead to a proof of Theorem 1 .

In this respect, given that the distribution of positive integers $n$ having a fixed number of prime factors $K<\kappa \log \log n$ is very well understood starting with the work of Landau and continuing with the work of Hardy and Ramanujan [6], it may seem that assertion $\mathbf{B}$ is more suitable for our purpose. However, this is not quite so either since most $n$ have $\omega(n)>(1-\varepsilon) \log \log n$ and for such numbers the lower bound on $P\left(2^{n}-1\right)$ given by $\mathbf{B}$ is only of the shape $\varphi(n)(\log n)^{1-(1-\varepsilon) \log 2}$, and this is not enough to guarantee the convergence of series (1) even with $\alpha=0$.

Conditionally, Murty and Wang [11] have shown that the $A B C$ conjecture implies that $P\left(2^{n}-1\right)>n^{2-\varepsilon}$ for all $\varepsilon>0$ once $n$ is sufficiently large with respect to $\varepsilon$. This certainly implies the conditional convergence of series (1) for all fixed $\alpha>0$. Murata and Pomerance [10] have proved, under the generalized Riemann hypothesis for various Kummerian fields, that the inequality $P\left(2^{n}-1\right)>n^{4 / 3} / \log \log n$ holds for almost all $n$, but they did not give explicit upper bounds on the size of the exceptional set either.

\section{Main tools}

As we have mentioned in Section 3, neither assertion A nor $\mathbf{B}$ of Section 3 is directly suitable for our purpose. However, another criterion, implicit in the work of Stewart [16] and which we present as Lemma 2 below (see also [10, Lemma 3]), plays an important role in our proof.

LEMMA 2. Let $n \geq 2$, and let $d_{1}<\cdots<d_{\ell}$ be all $\ell=2^{\omega(n)}$ square-free divisors of $n$. Then for all $n>6$,

$$
\#\left\{p \mid 2^{n}-1: p \equiv 1(\bmod n)\right\} \gg \frac{\log (2+\Delta(n) / \tau(n))}{\log \log P\left(2^{n}-1\right)},
$$

where

$$
\Delta(n)=\max _{i=1, \ldots, \ell-1} d_{i+1} / d_{i} .
$$

Stewart's [16] proof of Lemma 2 uses the original lower bounds for linear forms in logarithms of algebraic numbers due to Baker. It is interesting to note that following [16] (see also [10, Lemma 3]), but using instead the sharper lower bounds 
for linear forms in logarithms due to Matveev [9], does not seem to lead to any improvement of Lemma 2.

Let $1=d_{1}<d_{2}<\cdots<d_{\tau(n)}=n$ be all the divisors of $n$ and let

$$
\Delta_{0}(n)=\max _{i \leq \tau(n)-1} d_{i+1} / d_{i}
$$

Note that $\Delta_{0}(n) \leq \Delta(n)$.

We need the following result of Saias [13] on the distribution of positive integers $n$ with 'dense divisors'. Let

$$
\mathcal{G}(x, z)=\left\{n \leq x: \Delta_{0}(n) \leq z\right\}
$$

LEMMA 3. The bound

$$
\# \mathcal{G}(x, z) \asymp x \frac{\log z}{\log x}
$$

holds uniformly for $x \geq z \geq 2$.

Next we address the structure of integers with $\Delta_{0}(n) \leq z$. In what follows, as usual, an empty product is, by convention, equal to 1 .

LEMMA 4. Let $n=p_{1}^{e_{1}} \cdots p_{k}^{e_{k}}$ be the prime number factorization of a positive integer $n$, such that $p_{1}<\cdots<p_{k}$. Then $\Delta_{0}(n) \leq z$ if and only if, for each $i \leq k$, the inequality

$$
p_{i} \leq z \prod_{j<i} p_{j}^{e_{j}}
$$

holds.

PROOF. The necessity is clear since otherwise the ratio of the two consecutive divisors

$$
\prod_{j<i} p_{j}^{e_{j}} \quad \text { and } \quad p_{i}
$$

is larger than $z$.

The sufficiency can be proved by induction on $k$. Indeed, for $k=1$ it is trivial. By the induction assumption, we also have $\Delta_{0}(m) \leq z$, where $m=n / p_{1}^{e_{1}}$. Remarking that $p_{1} \leq z$, we also conclude that $\Delta_{0}(n) \leq z$.

\section{Proof of Theorem 1}

We put $\mathcal{E}=\left\{n: \tau(n) \geq(\log n)^{3}\right\}$. To bound \#E(x), let $x$ be large and $x /(\log x)^{2}<$ $n \leq x$. Since $n \in \mathcal{E}(x)$, we have that $\tau(n)>\left(\log \left(x /(\log x)^{2}\right)\right)^{3}>0.5(\log x)^{3}$ for all $x$ sufficiently large. Since

$$
\sum_{n \leq x} \tau(n)=O(x \log x)
$$


(see [7, Theorem 320]), we get that

$$
\# \mathcal{E}(x) \ll \frac{x}{(\log x)^{2}} .
$$

By the primitive divisor theorem (see [1], for example), there exists a prime factor $p \equiv 1(\bmod n)$ of $2^{n}-1$ for all $n>6$. Then, by partial summation,

$$
\begin{aligned}
\sum_{n \in \mathcal{E}(x)} \frac{(\log n)^{\alpha}}{P\left(2^{n}-1\right)} & \leq \sum_{n \in \mathcal{E}(x)} \frac{(\log n)^{\alpha}}{n} \leq 1+\int_{2}^{x} \frac{(\log t)^{\alpha}}{t} d \# \mathcal{E}(t) \\
& \leq 1+\frac{\# \mathcal{E}(x)}{x}+\int_{2}^{x} \frac{\# \mathcal{E}(t)(\log t)^{\alpha}}{t^{2}} d t \\
& \ll 1+\int_{2}^{x} \frac{d t}{t(\log t)^{2-\alpha}} \ll 1 .
\end{aligned}
$$

Hence,

$$
\sum_{n \in \mathcal{E}} \frac{(\log n)^{\alpha}}{P\left(2^{n}-1\right)}<\infty
$$

We now let $\mathcal{F}=\left\{n: P\left(2^{n}-1\right)>n(\log n)^{1+\alpha}(\log \log n)^{2}\right\}$. Clearly,

$$
\sum_{n \in \mathcal{F}} \frac{(\log n)^{\alpha}}{P\left(2^{n}-1\right)} \leq \sum_{n \geq 1} \frac{1}{n \log n(\log \log n)^{2}}<\infty .
$$

From now on, we assume that $n \notin \mathcal{E} \cup \mathcal{F}$. For a given $n$, we let

$$
\mathcal{D}(n)=\left\{d: d n+1 \text { is a prime factor of } 2^{n}-1\right\},
$$

and

$$
D^{+}(n)=\max \{d \in \mathcal{D}(n)\} .
$$

Since $P\left(2^{n}-1\right)=D^{+}(n) n+1$,

$$
D^{+}(n) \leq(\log n)^{1+\alpha}(\log \log n)^{2} .
$$

Assume that $L$ is large. Clearly, for $n \in\left[e^{L-1}, e^{L}\right], D^{+}(n) \leq L^{1+\alpha}(\log L)^{2}$. We let $\mathcal{H}_{d, L}$ be the set of $n \in\left[e^{L-1}, e^{L}\right]$ such that $D^{+}(n)=d$. We then note that by partial summation

$$
\begin{aligned}
S_{L} & =\sum_{\substack{e^{L-1} \leq n \leq e^{L} \\
n \notin \mathcal{E} \cup \mathcal{F}}} \frac{(\log n)^{\alpha}}{P\left(2^{n}-1\right)} \leq L^{\alpha} \sum_{d \leq L^{1+\alpha}(\log L)^{2}} \sum_{n \in \mathcal{H}_{d, L}} \frac{1}{n d+1} \\
& <\frac{L^{\alpha}}{e^{L-1}} \sum_{d \leq L^{1+\alpha}(\log L)^{2}} \frac{\# \mathcal{H}_{d, L}}{d} \ll \frac{L^{\alpha}}{e^{L}} \sum_{d \leq L^{1+\alpha}(\log L)^{2}} \frac{\# \mathcal{H}_{d, L}}{d} .
\end{aligned}
$$


We now estimate $\# \mathcal{H}_{d, L}$. We let $\varepsilon>0$ to be a small positive number depending on $\alpha$ which is to be specified later. We split $\mathcal{H}_{d, L}$ in two subsets as follows.

Let $\mathcal{I}_{d, L}$ be the set of $n \in \mathcal{H}_{d, L}$ such that

$$
\# \mathcal{D}(n)>M L^{\alpha+\varepsilon}(\log L)^{2},
$$

where $M$ is some positive integer depending on $\varepsilon$ to be determined later. Since $D^{+}(n) \leq L^{1+\alpha}(\log L)^{2}$, there exists an interval of length $L^{1-\varepsilon}$ which contains at least $M$ elements of $\mathcal{D}(n)$. Let them be $d_{0}<d_{1}<\cdots<d_{M-1}$. Write $k_{i}=d_{i}-d_{0}$ for $i=1, \ldots, M-1$. For fixed $d_{0}, k_{1}, \ldots, k_{M-1}$, by the Brun sieve (see, for example, [5, Theorem 2.3]),

$$
\begin{gathered}
\#\left\{n \in\left[e^{L-1}, e^{L}\right]: d_{i} n+1 \text { is a prime for all } i=1, \ldots, M\right\} \\
\ll \frac{e^{L}}{L^{M}} \prod_{p \mid d_{1} \cdots d_{M}}\left(1-\frac{1}{p}\right)^{-M} \ll \frac{e^{L}}{L^{M}}\left(\frac{\prod_{i=1}^{M} d_{i}}{\varphi\left(\prod_{i=1}^{M} d_{i}\right)}\right)^{M} \\
\ll \frac{e^{L}\left(\log \log \left(L^{3 M}\right)\right)^{M}}{L^{M}} \ll \frac{e^{L}(\log \log L)^{M}}{L^{M}},
\end{gathered}
$$

where we have used the fact that $\varphi(m) / m \gg 1 / \log \log y$ in the interval $[1, y]$ with $y=\left(L^{1+\alpha}(\log L)^{2}\right)^{M}<L^{3 M}$ (see [7, Theorem 328]). Summing the inequality (6) for all $d_{0} \leq L^{1+\alpha}(\log L)^{2}$ and all $k_{1}, \ldots, k_{M-1} \leq L^{1-\varepsilon}$, we get

$$
\# \mathcal{I}_{d, L} \ll \frac{e^{L}(\log L)^{M+2} L^{1+\alpha} L^{(M-1)(1-\varepsilon)}}{L^{M}}=\frac{e^{L}(\log L)^{M+2}}{L^{(M-1) \varepsilon-\alpha}} .
$$

We now choose $M$ to be the least integer such that $(M-1) \varepsilon>2+\alpha$, and with this choice of $M$ we get that

$$
\# \mathcal{I}_{d, L} \ll \frac{e^{L}}{L^{2}} .
$$

We now deal with the set $\mathcal{J}_{d, L}$ consisting of the numbers $n \in \mathcal{H}_{d, L}$ with $\# \mathcal{D}(n) \leq$ $M L^{\alpha+\varepsilon}(\log L)^{2}$. To these, we apply Lemma 2. Since $\tau(n)<(\log n)^{3}$ and $P\left(2^{n}-\right.$ 1) $<n^{2}$ for $n \in \mathcal{H}_{d, L}$, Lemma 2 yields

$$
\log \Delta(n) / \log \log n \ll \# \mathcal{D}(n) \ll L^{\alpha+\varepsilon}(\log L)^{2} .
$$

Thus,

$$
\log \Delta(n) \ll L^{\alpha+\varepsilon}(\log L)^{3}
$$

Therefore

$$
\Delta_{0}(n) \leq \Delta(n) \leq z_{L},
$$

where

$$
z_{L}=\exp \left(c L^{\alpha+\varepsilon}(\log L)^{3}\right)
$$

and $c>0$ is some absolute constant. 
We now further split $\mathcal{J}_{d, L}$ into two subsets. Let $\mathcal{S}_{d, L}$ be the subset of $n \in \mathcal{J}_{d, L}$ such that $P(n)<e^{L / \log L}$. From known results concerning the distribution of smooth numbers (see the corollary to [2, Theorem 3.1], or [8, 17], for example),

$$
\# \mathcal{S}_{d, L} \leq \frac{e^{L}}{L^{(1+o(1)) \log \log L}} \ll \frac{e^{L}}{L^{2}} .
$$

Let $\mathcal{T}_{d, L}=\mathcal{J}_{d, L} \backslash \mathcal{S}_{d, L}$. For $n \in \mathcal{T}_{d, L}$, we have $n=q m$, where $q>e^{L / \log L}$ is a prime. Fix $m$. Then $q<e^{L} / m$ is a prime such that $q d m+1$ is also a prime. By the Brun sieve again,

$$
\begin{aligned}
\#\{q & \left.\leq e^{L} / m: q, q d m+1 \text { are primes }\right\} \\
& \ll \frac{e^{L}}{m\left(\log \left(e^{L} / m\right)\right)^{2}}\left(\frac{m d}{\varphi(m d)}\right) \ll \frac{e^{L}(\log L)^{3}}{L^{2} m},
\end{aligned}
$$

where in the above inequality we used the minimal order of the Euler function in the interval $\left[1, e^{L} L^{1+\alpha}(\log L)^{2}\right]$ together with the fact that

$$
\log \left(e^{L} / m\right) \geq \frac{L}{\log L} .
$$

We now sum estimate (10) over all the allowable values for $m$.

An immediate consequence of Lemma 4 is that since $\Delta_{0}(n) \leq z_{L}$, then $\Delta_{0}(m) \leq z_{L}$ for $m=n / P(n)$. Thus, $m \in \mathcal{G}\left(e^{L}, z_{L}\right)$. Using Lemma 3 and partial summation, we immediately get

$$
\begin{aligned}
\sum_{m \in \mathcal{G}\left(e^{L}, z_{L}\right)} \frac{1}{m} & \leq \int_{2}^{e^{L}} \frac{d\left(\# \mathcal{G}\left(t, z_{L}\right)\right)}{t} \leq \frac{\# \mathcal{G}\left(e^{L}, z_{L}\right)}{e^{L}}+\int_{2}^{e^{L}} \frac{\# \mathcal{G}\left(t, z_{L}\right)}{t^{2}} d t \\
& \ll \frac{\log z_{L}}{L}+\log z_{L} \int_{2}^{e^{L}} \frac{d t}{t \log t} \\
& \ll \log z_{L} \log L \ll L^{\alpha+\varepsilon}(\log L)^{4}
\end{aligned}
$$

Thus,

$$
\# \mathcal{T}_{d, L} \ll \frac{e^{L}(\log L)^{3}}{L^{2}} \sum_{m \in \mathcal{M}_{d, L}} \frac{1}{m} \ll \frac{e^{L}(\log L)^{7} L^{\alpha+\varepsilon}}{L^{2}}<\frac{e^{L}}{L^{2-\alpha-2 \varepsilon}},
$$

when $L$ is sufficiently large. Combining estimates (8), (9) and (11), we get that

$$
\# \mathcal{H}_{d, L} \leq \# \mathcal{I}_{d, L}+\# \mathcal{S}_{d, L}+\# \mathcal{T}_{d, L} \ll \frac{e^{L}}{L^{2-\alpha-2 \varepsilon}} .
$$

Thus, returning to series (5), we get that

$$
S_{L} \leq \sum_{d \leq L^{1+\alpha}(\log L)^{2}} \frac{1}{L^{2-2 \alpha-2 \varepsilon}} \ll \frac{\log L}{L^{2-2 \alpha-2 \varepsilon}} .
$$


Since $\alpha<1 / 2$, we can choose $\varepsilon>0$ such that $2-2 \alpha-2 \varepsilon>1$ and then the above arguments show that

$$
\sum_{n \geq 1} \frac{(\log n)^{\alpha}}{P\left(2^{n}-1\right)} \ll 1+\sum_{L} \frac{\log L}{L^{2-2 \alpha-\varepsilon}}<\infty
$$

which is the desired result.

\section{References}

[1] G. D. Birkhoff and H. S. Vandiver, 'On the integral divisors of $a^{n}-b^{n}$ ', Ann. of Math. (2) 5 (1904), 173-180.

[2] E. R. Canfield, P. Erdôs and C. Pomerance, 'On a problem of Oppenheim concerning "factorisatio numerorum" ', J. Number Theory 17 (1983), 1-28.

[3] P. Erdôs, P. Kiss and C. Pomerance, 'On prime divisors of Mersenne numbers', Acta Arith. 57 (1991), 267-281.

[4] P. Erdôs and T. N. Shorey, 'On the greatest prime factor of $2^{p}-1$ for a prime $p$ and other expressions', Acta Arith. 30 (1976), 257-265.

[5] H. Halberstam and H.-E. Richert, Sieve Methods (Academic Press, London, 1974).

[6] G. H. Hardy and S. Ramanujan, 'The normal number of prime factors of an integer', Quart. J. Math. (Oxford) 48 (1917), 76-92.

[7] G. H. Hardy and E. M. Wright, An Introduction to the Theory of Numbers, 5th edn (Clarendon Press, Oxford, 1979).

[8] A. Hildebrand and G. Tenenbaum, 'Integers without large prime factors', J. de Théorie des Nombres de Bordeaux 5 (1993), 411-484.

[9] E. M. Matveev, 'An explicit lower bound for a homogeneous rational linear form in logarithms of algebraic numbers II', Izv. Ross. Akad. Nauk. Ser. Math. 64 (2000), 125-180; Engl. transl. Izv. Math. 64 (2000), 1217-1269.

[10] L. Murata and C. Pomerance, On the Largest Prime Factor of a Mersenne Number, Number Theory CRM Proceedings Lecture Notes, 36 (American Mathematical Society, Providence, RI, 2004), pp. 209-218.

[11] R. Murty and S. Wong, 'The $A B C$ conjecture and prime divisors of the Lucas and Lehmer sequences', in: Number Theory for the Millennium, III, Urbana, IL, 2000 (A K Peters, Natick, MA, 2002), pp. 43-54.

[12] C. Pomerance, 'On primitive divisors of Mersenne numbers', Acta Arith. 46(4) (1986), 355-367.

[13] E. Saias, 'Entiers à diviseurs denses 1', J. Number Theory 62 (1997), 163-191.

[14] A. Schinzel, 'On primitive prime factors of $a^{n}-b^{n}$, Proc. Cambridge Philos. Soc. 58 (1962), $555-562$.

[15] C. L. Stewart, 'The greatest prime factor of $a^{n}-b^{n}$ ', Acta Arith. 26(4) (1974/75), 427-433.

[16] _ 'On divisors of Fermat, Fibonacci, Lucas and Lehmer numbers', Proc. London Math. Soc. 35(3) (1977), 425-447.

[17] G. Tenenbaum, Introduction to Analytic and Probabilistic Number Theory (Cambridge University Press, Cambridge, 1995).

KEVIN FORD, Department of Mathematics, The University of Illinois at Urbana-Champaign, Urbana, Champaign, IL 61801, USA

e-mail: ford@math.uiuc.edu 
FLORIAN LUCA, Instituto de Matemáticas,

Universidad Nacional Autónoma de México, C.P. 58089, Morelia, Michoacán, México e-mail: fluca@matmor.unam.mx

IGOR E. SHPARLINSKI, Department of Computing, Macquarie University, Sydney, NSW 2109, Australia

e-mail: igor@ics.mq.edu.au 